\title{
Research on Emergency Logistics Management by Employing Uncertain Case-Based Reasoning
}

\author{
Xuedou $\mathrm{Yu}^{1, *}$ and Xiulian $\mathrm{Gao}^{2}$ \\ ${ }^{1}$ College of Information Management, Dezhou University, Dezhou, Shandong, China \\ ${ }^{2}$ College of Mathematical Science, Dezhou University, Dezhou, China \\ ${ }^{*}$ Corresponding author
}

\begin{abstract}
A new solution strategy about emergency logistics by employing uncertain case-based reasoning was provided for the management of emergency logistics. A scientific case-based reasoning rule and model reasoning algorithm were established. According to expert opinions, the membership function of each attribute of factors affecting logistics can be obtained. Then, according to the uncertain inference theory, the similar degree of new case and every known case can be calculated, and the similar degree are denoted as s1, s2, ,-, sk, respectively. Through these similar degrees, the ownership of the new cases can be determined. Through examples, the classified method is correct and effective.
\end{abstract}

Keywords - case-based reasoning; uncertain theory; emergency logistics; similar degree

\section{INTRODUCTION}

Now, China's "One Belt and One Road" provides new opportunities to strengthen the international division of labor and cooperation for the development of the world economy, at the same time, the importance of logistics is further apparent, logistics efficiency has become a hot topic of current research. To enhance the efficiency of logistics, the modern logistics must be relied on. Modern logistics can carry out logistics transportation and improve people's living standard with maximum benefit. But the natural disasters and the emergence of unexpected events to modern logistics has brought a test, how to improve this management capacity is the subject of our research.

Case-based reasoning (CBR) theory has been widely applied in the field of artificial intelligence since the theory was put forward, whose main idea is to solve the proposing problem by using successful solutions of the past problems, and the current problem solution can be obtained through modifying related parameters.

The advantages of CBR are that the system does not need a large number of cases at the beginning, just need to save some successful or unsuccessful cases in the system. According to the similar degree of the new case with existing cases, we can determine the ownership of the new case. A lot of scholars have taken deep research on CBR. Such as Jianyuan Yan, Peggy E.Chaudhry and Sohail S.Chaudhry proposed a casebased reasoning model framework for a 3PL evaluation and selection system; Shi F, Xu J, Sun S proposed a defined similarity measurement model based on FCBR. But most of them study it by employing fuzzy theory [4-10,11,14,16-19], however, most attributes of problem cannot be described by fuzzy data.

In this paper, the author presents a new model about emergency logistics by using uncertain case-based reasoning (UCBR) technologies.

\section{PRELIMINARIES}

\section{A. Uncertainty Space}

Definition 1 (Liu [1]) Let $\Gamma$ be a nonempty set, let $L$ be a $\sigma$-algebra over $\Gamma$, and let $\mathrm{M}$ be an uncertain measure, then the triplet $(\Gamma, \mathrm{L}, \mathrm{M})$ is called an uncertainty space.

In order to present an axiomatic definition of uncertain measure, it is necessary to assign to each event $\Lambda$ a number $\mathrm{M}\{\Lambda\}$ which indicates the level that $\Lambda$ will occur. In order to rationally deal with belief degrees, Liu[1] suggested the following three axioms:

Axiom 1. (Normality Axiom) $\mathrm{M}\{\Gamma\}=1$ for the universal set $\Gamma$.

Axiom 2 (Duality Axiom) $M\{\Lambda\}+M\left\{\Lambda^{c}\right\}=1$ for any event $\Lambda$.

Axiom 3 (Subadditivity Axiom) For every countable sequence of events $\left\{\Lambda_{\mathrm{i}}\right\}$, we have

$$
\mathrm{M}\left\{\mathrm{U}_{i-1}^{\infty} \Lambda_{i}\right\} \leq \sum_{i=1}^{\infty} \mathrm{M}\left\{\Lambda_{i}\right\}
$$

Denition 2 (Liu [1]) The set function $M$ is called an uncertain measure if it satisfies the normality, duality, and subadditivity axioms.

Theorem 1 (Monotonicity Theorem) The uncertain measure is a monotone increasing set function. That is, for any events $\Lambda_{1}$ and $\Lambda_{2}$ with $\Lambda_{1} \in \Lambda_{2}$, we have

$$
\mathrm{M}\left\{\Lambda_{1}\right\} \leq \mathrm{M}\left\{\Lambda_{2}\right\}
$$

\section{B. Uncertain Variables}

Definition 3 (Liu [1]) An uncertain variable is a measurable function $\xi$ from an uncertainty $\operatorname{space}(\Gamma, \mathrm{L}, \mathrm{M})$ to the set of real numbers, i.e., for any Borel set B of real numbers, the set 


$$
\{\xi \in \mathrm{B}\}=\{\gamma \in \Gamma \mid \xi(\gamma) \in \mathrm{B}\}
$$

is an event.

Definition 4 An n-dimensional uncertain vector is a measurable function from an uncertainty $\operatorname{space}(\Gamma, \mathrm{L}, \mathrm{M})$ to the set of n-dimensional real vectors, i.e., for any Borel set B of $\mathrm{R}^{n}$, the set

$$
\{\xi \in \mathrm{B}\}=\{\gamma \in \Gamma \mid \xi(\gamma) \in \mathrm{B}\}
$$

is an event.

\section{Identification Function}

Definition 5 (Liu [1]) An uncertain variable $\xi$ is said to have a first identification function $\lambda$ if

(i) $\lambda(x)$ is a nonnegative function on $\mathrm{R}$ such that

$$
\sup (\lambda(x)+\lambda(y))=1
$$

(ii) for any set $\mathrm{B}$ of real numbers, we have

$$
M\{\xi \in \mathrm{B}\}=\left\{\begin{array}{c}
\sup _{x \in \mathrm{B}}^{\lambda(x), \text { if } \sup \lambda(x)<0.5} \underset{x \in \mathrm{B}}{1-\sup _{x \in \mathrm{B}^{\mathrm{c}}} \lambda(x), \text { if } \sup _{x \in \mathrm{B}} \lambda(\underset{x}{x})<0.5}
\end{array}\right\}
$$

\section{Expected Value}

Definition 6 Let $\xi$ be an uncertain variable. Then the expected value of $\xi$ is defined by

$$
\mathrm{E}[\xi]=\int_{0}^{+\infty} M\{\xi \geq \mathrm{r}\} \mathrm{dr}-\int_{-\infty}^{0} M\{\xi \leq \mathrm{r}\} \mathrm{dr}
$$

provide that at least one of the two integrals is finite.

\section{E. Uncertain Set}

Uncertain set theory was proposed by Liu[3] in 2010 as a generalization of uncertainty theory to the domain of uncertain sets.

Definition 7 An uncertain set is a measurable function $\xi$ from an uncertainty $\operatorname{space}(\Gamma, \mathrm{L}, \mathrm{M})$ to a collection of sets of real numbers, i.e., for any Borel set $\mathrm{B}$ of real numbers, the set

$$
\{\xi \subset \mathrm{B}\}=\{\gamma \grave{o} \Gamma \mid \xi(\gamma) \subset \mathrm{B}\}
$$

is an event.

Definition 8 Let $\xi$ and $\eta$ be two uncertain sets on the uncertainty space $(\Gamma, \mathrm{L}, \mathrm{M})$. Then the union $\xi \cup \eta$ of uncertain sets $\xi$ and $\eta$ is

$$
(\xi \cup \eta)(\gamma)=\xi(\gamma) \cup \eta(\gamma), \forall \gamma \in \Gamma
$$

Then intersection $\xi \cap \eta$ of uncertain sets ${ }^{\xi}$ and $\eta$ is

$$
(\xi \cap \eta)(\gamma)=\xi(\gamma) \cap \eta(\gamma), \forall \gamma \in \Gamma
$$

The complement $\xi^{\mathrm{c}}$ of uncertain set $\xi_{\text {is }}$

$$
\xi^{\mathrm{c}}(\gamma)=\xi(\gamma)^{\mathrm{c}}, \forall \gamma \in \Gamma
$$

Definition 9 The uncertain sets $\xi_{1}, \xi_{2}, \mathrm{~L}, \xi_{m}$ are said to be independent if

$$
\mathrm{M}\left\{\mathrm{I}_{i=1}^{m}\left(\xi_{i} \subset \mathrm{B}_{i}\right)\right\}=\min _{1 \leq i \leq m} \mathrm{M}\left\{\xi_{i} \subset \mathrm{B}_{i}\right\}
$$

and

$$
\mathrm{M}\left\{\mathrm{U}_{i=1}^{m}\left(\xi_{i} \subset \mathrm{B}_{i}\right)\right\}=\min _{1 \leq i \leq m} \mathrm{M}\left\{\xi_{i} \subset \mathrm{B}_{i}\right\}
$$

for any Borel sets $B_{1}, B_{2}, L, B_{m}$ of real numbers.

Definition 10 An uncertain set $\xi$ is said to have a membership function $\mu$ if for any Borel set B of real numbers, we have

$$
\begin{aligned}
& \mathrm{M}\{\mathrm{B} \subset \xi\}=\inf _{x \in \mathrm{B}} \mu(\mathrm{x}) \\
& \mathrm{M}\{\xi \subset \mathrm{B}\}=1-\sup _{x \in \mathrm{B}^{c}} \mu(\mathrm{x})
\end{aligned}
$$

\section{F. Uncertain Inference}

Inference Rule 1 (Liu[3]) Let $X$ and $Y$ be two concepts. Assume a rule "if $\mathrm{X}$ is an uncertain set $\xi$ then $\mathrm{Y}$ is an uncertain set $\eta$ ". From $\mathrm{X}$ is a constant $a$ we infer that $\mathrm{Y}$ is an uncertain set

$$
\eta^{*}=\left.\eta\right|_{a \in \xi}
$$

which is the conditional uncertain set $\eta$ given $a \in \xi$.

Theorem 2 (Liu[3]) In Inference Rule 1, if $\xi$ and $\eta$ are independent uncertain sets with membership functions $\mu$ and $v$, respectively, If $\xi^{*}$ is a constant $a$, then the inference rule 1 yields that $\eta^{*}$ has a membership function

$$
v^{*}(y)= \begin{cases}v(y) / \mu(a), & \text { if } v(y)<\mu(a) / 2 \\ (v(y)+\mu(a)-1) / \mu(a), & \text { if } v_{i}(y)>1-\mu(a) / 2 \\ 0.5, & \text { otherwise }\end{cases}
$$


Inference Rule 2 (Gao-Gao-Ralescu[13]) makes $\mathrm{X}_{1}, \mathrm{X}_{2}, \mathrm{~K}, \mathrm{X}_{m}$ be concepts. Assume rules "if $\mathrm{X}_{1}$ is $\xi_{i 1}$ and $\mathrm{L}$ and $\mathrm{X}_{m}$ is $\xi_{i m}$ then $\mathrm{Y}$ is $\eta_{i}$ " for $\mathrm{i}=1,2, \mathrm{~L}, \mathrm{k}$. From $\mathrm{X}_{1}$ is $a_{1}$ and n and $\mathrm{X}_{m}$ is $a_{m}$ we infer that $\mathrm{Y}$ is an uncertain set

$$
\eta^{*}=\sum_{i=1}^{k} \frac{\left.c_{i} \mathrm{~g} \eta_{i}\right|_{\left(a_{1} \in \xi_{i 1}\right) \cap\left(a_{2} \in \xi_{i 2}\right) \frown \mathrm{L}\left(a_{m} \in \xi_{i m}\right)}}{c_{1}+c_{2}+\mathrm{L}+c_{k}}
$$

where the coefficients are determined by $c_{i}=\mathrm{M}\left\{\left(a_{1} \in \xi_{i 1}\right) \cap\left(a_{2} \in \xi_{i 2}\right) \cap \mathrm{L}\left(a_{m} \in \xi_{\text {im }}\right)\right\}$, for $\mathrm{i}=1,2, \mathrm{~L}, \mathrm{k}$.

Theorem 3 (Gao-Gao-Ralescu[13]) Assume $\xi_{i 1}, \xi_{i 2}, \mathrm{~L}, \xi_{i m}, \eta_{i}$ are independent uncertain sets with membership functions of $\mu_{i 1}, \mu_{i 2}, \mathrm{~L}, \mu_{i m}, v_{i}, \mathrm{i}=1,2 \mathrm{~L}, \mathrm{k}$ respectively. If $\xi_{1}^{*}, \xi_{2}^{*}, \mathrm{~L}, \xi_{m}^{*}$ are constanted with $a_{1}, a_{2}, \mathrm{~L}, a_{m}$, respectively, then the inference rule 2 yields

$$
\eta^{*}=\sum_{i=1}^{k} \frac{c_{i} \mathrm{~g}_{i}^{*}}{c_{1}+c_{2}+\mathrm{L}+c_{k}}
$$

where $\eta^{*}$ are uncertain sets whose membership functions are given by

$$
v_{i}^{*}(y)=\left\{\begin{array}{lc}
v_{i}(y) / c_{i}, & \text { if } v_{i}(y)<c_{i} / 2 \\
\left(v_{i}(y)+c_{i}-1\right) / c_{i}, & \text { if } v_{i}(y)>1-c_{i} / 2 \\
0.5, & \text { otherwise }
\end{array}\right.
$$

and $c_{i}$ are constants determined by $c_{i}=\min _{1 \leq l \leq m} \mu_{i l}\left(a_{l}\right)$ for $i=1,2, \mathrm{~L}, \mathrm{k}$, respectively.

\section{INFLUENCE FACTORS OF EMERGENCY LOGISTICS}

In emergency situations, in order to meet the urgent needs of the victims, we enable the process of planning, managing and controlling the effective flow of the relief supplies, information and services from the origin to the destination point. [20]

In order to manage the emergency logistics, we need to understand the factors that affect the logistics. According to the experts, the basic elements, safeguard factors, material elements, environmental factors and functional factors have an impact on the results of emergency logistics. The casebased reasoning model established based on these factors.

\section{CASE-BAsed ReAsoning Model about EMERGENCY LOGISTICS}

Case-based reasoning model about emergency logistics mainly includes the case expression model, the storage organization and the similar degree retrieval model. The flow chart of the case-based reasoning model about emergency logistics is shown in Figure I.

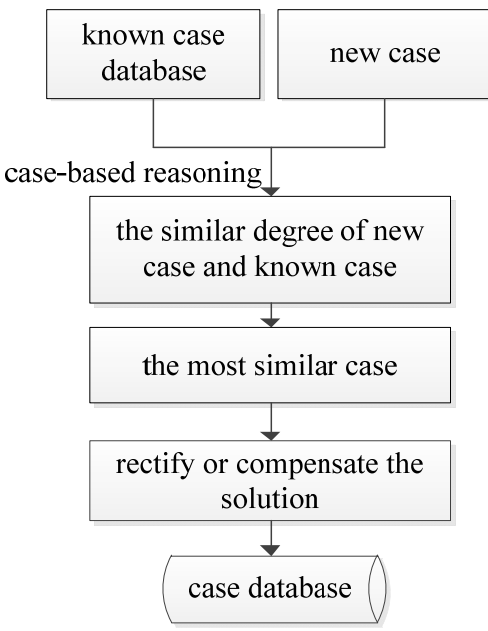

FIGURE I. FLOW CHART OF THE CASE-BASED REASONING MODEL ABOUT EMERGENCY LOGISTICS

\section{A. Case Expression Model about Emergency Logistics}

Case expression model about emergency logistics consists of the main factors affecting the emergency logistics. When an emergency logistics occurs, we need to extract all the attribute values that correspond to it. The basic expressions of the attribute characteristics in the case database is as follows.

$$
\begin{gathered}
\mathrm{X}_{i}=\left(\xi_{i 1}, \xi_{i 2}, \mathrm{~L}, \xi_{i n}\right) \\
\mathrm{X}_{i}(\mathrm{i}=1,2, \mathrm{~L}, \mathrm{k}) \\
i ; \xi_{i j}(\mathrm{i}=1,2, \mathrm{~L}, \mathrm{k}, \mathrm{j}=1,2, \mathrm{~L}, \mathrm{n}) \text { expresses the characteristic } j \text { of }
\end{gathered}
$$
case $i$.

\section{B. Storage Organization of Emergency Logistics Case Database}

According to the rules of the database creation, we build the case database. In order to improve the case retrieval performance, we build the index aiming at the corresponding fields in the database. The storage organization of process case database is shown in Figure II. The expression of the known case and its solution is as follows.

$$
\mathrm{X}_{i}=\left(\xi_{i 1}, \xi_{i 2}, \mathrm{~L}, \xi_{i n}, \gamma_{i 1}, \gamma_{i 2}, \mathrm{~L}, \gamma_{i m}\right)
$$

$\mathrm{X}_{i}(\mathrm{i}=1,2, \mathrm{~L}, \mathrm{k}) \quad$ expresses the case $i$; $\xi_{i j}(\mathrm{i}=1,2, \mathrm{~L}, \mathrm{k}, \mathrm{j}=1,2, \mathrm{~L}, \mathrm{n})$ expresses the characteristic $j$ of case $i, \quad \gamma_{i l}(\mathrm{i}=1,2, \mathrm{~L}, \mathrm{k}, 1=1,2, \mathrm{~L}, \mathrm{~m}) \quad$ expresses the solution characteristic $l$ of case $i$. 


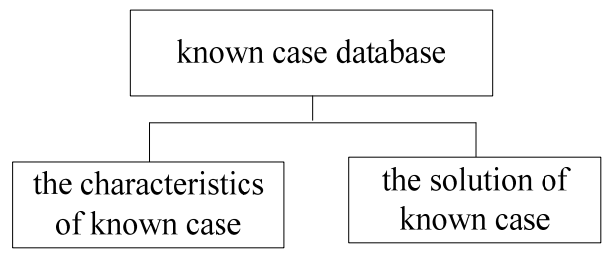

FIGURE II. ORGANIZATION CHART OF STORAGE ORGANIZATION OF EMERGENCY LOGISTICS CASE DATABASE

\section{Similar Degree Retrieval Model of Emergency Logistics Case Database}

Emergency logistics similar degree retrieval is based on the characteristics of the new case. According to the formula (16-20), we can obtain the similar degree s1, s2, L , sk between the new case and the known cases in database. By comparison, we pick up the largest variable, assume the biggest variable is si. So we can obtain the most similar case i. the solution of case $i$ is the solution of new case. If the solution of the new case is not reasonable, it is necessary to modify them according to the domain knowledge to complete the retrieval and case reasoning by employing UCBR. The results obtained directly or the revised results are stored in the known case database according to formula (22). The retrieval flow chart of case-based reasoning by employing UCBR is shown in Figure III.

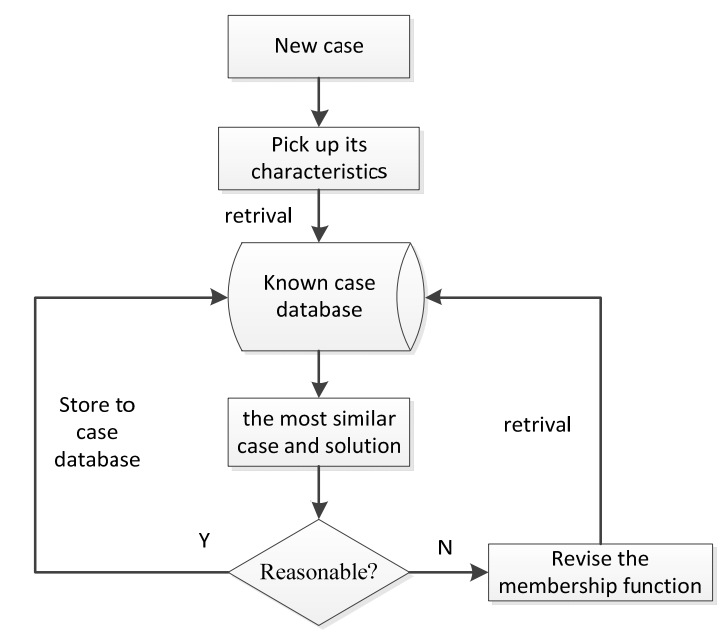

FIGURE III. RETRIEVAL FLOW CHART OF CASE-BASED REASONING BY EMPLOYING UCBR

\section{CASE REASONING EXPERIMENT IN EMERGENCY LOGISTICS}

In order to verify the validity and the correctness of the application of emergency logistics by employing UCBR, the following experiments are designed.

Step 1, according to expert opinion, we assign the corresponding value to the attributes of known case.

Step 2, according to the characteristics of the new case and expert opinions, we determine the membership function of each attribute.
Step 3, according to the formula (16-20), to calculate each similar degree $\mathrm{si}(\mathrm{i}=1,2, \mathrm{~L}, \mathrm{k})$ between the new case and each known case in known case database.

Step 4, acquire the biggest similar degree $\operatorname{si}(\mathrm{i}=1,2, \mathrm{~L}, \mathrm{k})$ by calculation. According to the formula (21), we can obtain the solution. If the solution of the new case is not reasonable, turn to Step 5.

Step 5, to modify the membership function of each attribute according to the domain knowledge, return to Step 3.

According to the algorithm, we can easily obtain the biggest similar degree $\mathrm{si}(\mathrm{i}=1,2, \mathrm{~L}, \mathrm{k})$. In following, we give an example.

Through questionnaire by experts, we can obtain several common characteristics and their weight, as shown below [15]

(1) Organization mechanism. (2) Materials acquisition. (3) Construction of emergency logistics center. (4) Personnel management of emergency logistics. (5) Material reserve and management. (6) Transportation of emergency supplies. (7) Distribution of emergency supplies. (8) Weather conditions. (9) Road conditions.

Through questionnaire by experts, we can obtain the weight of each factor above as the follows.

Assuming that $\mathrm{X}_{1}, \mathrm{X}_{2}$ is the known case and $\mathrm{Y}_{1}$ is the new case:

$$
\begin{gathered}
\mathrm{X}_{1}=\left(\xi_{11}, \xi_{12}, \xi_{13}, \xi_{14}, \xi_{15}, \xi_{16}, \xi_{17}, \xi_{18}, \xi_{19}\right) \\
\mathrm{X}_{2}=\left(\xi_{21}, \xi_{22}, \xi_{23}, \xi_{24}, \xi_{25}, \xi_{26}, \xi_{27}, \xi_{28}, \xi_{29}\right) \\
\mathrm{Y}_{1}=\left(\eta_{11}, \eta_{12}, \eta_{13}, \eta_{14}, \eta_{15}, \eta_{16}, \eta_{17}, \eta_{18}, \eta_{19}\right)
\end{gathered}
$$

So, the membership function of each attribute in $\mathrm{X}_{1}, \mathrm{X}_{2}$ and $Y_{1}$ as follows.

$$
\begin{gathered}
\mu_{11}=0.7, \mu_{12}=0.75, \mu_{13}=0.85, \mu_{14}=0.65, \mu_{15}=0.65, \\
\mu_{16}=0.75, \mu_{17}=0.8, \mu_{18}=0.9, \mu_{19}=0.75 \\
\mu_{21}=0.8, \mu_{22}=0.85, \mu_{23}=0.65, \mu_{24}=0.55, \mu_{25}=0.75, \\
\mu_{26}=0.85, \mu_{27}=0.7, \mu_{28}=0.75, \mu_{29}=0.95 \\
v_{11}=0.6, v_{12}=0.3, v_{13}=0.2, v_{14}=0.5, v_{15}=0.3, \\
v_{16}=0.2, v_{17}=0.2, v_{18}=0.8, v_{19}=0.3
\end{gathered}
$$

According to the algorithm, we compute the similar degree s1 and s2, respectively.

The similar degree $\mathrm{s} 1$ between $\mathrm{X}_{1}$ and $\mathrm{Y}_{1}$ is computed according to the formula (16-20). 


$$
\begin{gathered}
\eta_{11}^{*}=0.5, \eta_{12}^{*}=0.4, \eta_{13}^{*}=0.31, \eta_{14}^{*}=0.5, \eta_{15}^{*}=0.46, \\
\eta_{16}^{*}=0.27, \eta_{17}^{*}=0.29, \eta_{18}^{*}=0.55, \eta_{19}^{*}=0.4 \\
s 1=\eta_{1}^{*}=0.428
\end{gathered}
$$

The similar degree s2 between $X_{2}$ and $Y_{1}$ is computed according to the formula (16-20).

$$
\begin{gathered}
\eta_{21}^{*}=0.5, \eta_{22}^{*}=0.5, \eta_{23}^{*}=0.5, \eta_{24}^{*}=0.5, \eta_{25}^{*}=0.5, \\
\eta_{26}^{*}=0.5, \eta_{27}^{*}=0.5, \eta_{28}^{*}=0.5, \eta_{29}^{*}=0.5 \\
s 2=\eta_{2}^{*}=0.5
\end{gathered}
$$

$\mathrm{Y}_{1}$ is a similar case about $\mathrm{X}_{2}$ due to $\mathrm{s} 1<\mathrm{s} 2$.

\section{CONCLUSION}

The case-based reasoning model is based on the uncertainty theory in the management of emergency logistics. This method makes full use of the human perception in emergency logistics by the relationship between the new case and historical data. Experiments show that the reasoning way of emergency logistics by employing UCBR is correct and effective.

\section{ACKNOWLEDGMENTS}

Supported by project grants from Shandong Province Nature Science Fund (ZR2013GL001) and Dezhou soft science research plan project (NO.43 (2013)). All authors have no conflict of interest in this paper. This article does not contain any studies with human participants or animals performed by any of the authors.

\section{REFERENCES}

[1] Liu B:' Uncertainty Theory, 2nd ed'( Springer-Verlag, Berlin, 2007)

[2] Liu, Y:' Uncertain random variables: a mixture of uncertainty and randomness'. Soft Compute, 2013, 17(4), pp 625-634.

[3] Liu B:' Uncertain set theory and uncertain inference rule with application to uncertain control'. Journal of Uncertain Systems, 2010, 4(2), pp 83-98.

[4] Negar Armaghan, Jean Renaud:'An application of multi-criteria decision aids models for Case-Based Reasoning'. Information Sciences, 2012, 210,pp 55-66.

[5] M. Salam'o andM. L'opez-S'anchez:'Rough set based approaches to feature selection for Case-Based Reasoning classifiers'. Pattern Recognition Letters, 2011, 32(2), pp 280-292.

[6] Fuqian Shi, Jiang Xu, and Shouqian Sun:' Fuzzy Case-Based Reasoning in Product Style Acquisition Incorporating Valence-Arousal-Based Emotional Cellular Model'. Journal of Applied Mathematics, 2012, 2012(1110-757X),pp 155-172.

[7] S. T. Li and H. F. Ho:'Predicting financial activity with evolutionary fuzzy case-based reasoning'. Expert Systems with Applications, 2009, 36(1),pp 411-422.

[8] P. C. Chang, C. Y. Fan, and W. Y. Dzan:' A CBR-based fuzzy decision tree approach for database classification'. Expert Systems with Applications, 2010, 37(1), pp 214-225.
[9] Y. J. Jiang, J. Chen, and X. Y. Ruan:' Fuzzy similarity-based rough set method for case-based reasoning and its application in tool selection'. International Journal of Machine Tools and Manufacture, 2006, 46(2), pp 107-113.

[10] T. Y. Slonim and M. Schneider:' Design issues in fuzzy case-based reasoning'. Fuzzy Sets and Systems, 2001, 117(2),pp 251-267.

[11] Jianyuan Yan, Peggy E. Chaudhry and Sohail S. Chaudhry:' A model of a decision support system based on case-based reasoning for third-party logistics evaluation'. Expert Systems, 2010, 20(4),pp 196-207

[12] Liu, B:' Some research problems in uncertainty theory'. Journal of Uncertain Systems, 2009, 3(1), pp 3-10.

[13] Gao X, Gao Y, and Ralescu DA:' On Liu's inference rule for uncertain systems'. International Journal of Uncertainty, Fuzziness and Knowledge-Based Systems, 2010, 18(1),pp 1-11.

[14] Negar Armaghan, Jean Renaud:' An application of multi-criteria decision aids models for Case-Based Reasoning'. Information Sciences, 2012, 210(2012), pp 55-66

[15] Hua Ming, Cheng Lei:' Research on Influencing Factors of Emergency Logistics Based on the Interpretative Structural Model'. Logistics SciTech, 2009(5), pp 117-119

[16] P Berka:' NEST: A Compositional Approach to Rule-Based and CaseBased Reasoning'. Advances in Artificial Intelligence, 2011(16877470):4.

[17] Peterson, M. R., Young, R. R., \& Gordon, G. A:'The application of supply chain management principles to emergency management logistics: An empirical study'. Journal of Emergency Management, 2016, 14(4),pp 245-58

[18] Douglas Alem, Alistair Clark, Alfredo Moreno:' Stochastic network models for logistics planning in disaster relief'. European Journal of Operational Research, 2016, 255(1), pp 187-206

[19] Sheu, J. B:' Challenges of emergency logistics management'. Transportation Research Part E Logistics \& Transportation Review, 2007, 43(6), pp 655-659 